\title{
Inflammation in Peritoneal Dialysis
}

\author{
Kar Neng Lai Joseph C.K. Leung \\ Division of Nephrology, Department of Medicine, Queen Mary Hospital, University of Hong Kong, \\ Hong Kong, SAR, China
}

\section{Key Words}

Adipocytes · Fibrosis • Inflammation • Mesothelium •

Peritoneal dialysis

\begin{abstract}
During peritoneal dialysis, peritoneal cells are repeatedly exposed to a non-physiological hypertonic environment with high glucose content and low pH. Current sterile dialysis solutions cause inflammation in the submesothelial compact zone that leads to fibrosis, neoangiogenesis, progressive increases in solute transfer and even ultrafiltration failure. The peritoneal dysfunction will further be amplified with the development of an epithelial-to-mesenchymal transition of mesothelial cells and the dissipation of the osmotic driving force through increased area and solute transport that accompanied neoangiogenesis of the submesothelial microvasculature. The alteration in the peritoneal membrane will further be aggravated by peritonitis, advanced glycation end-products and glucose degradation products. Finally, there are emerging new data supporting a pro-inflammatory role of peritoneal adipocytes.
\end{abstract}

Copyright $\odot 2010$ S. Karger AG, Basel

\section{Introduction}

The peritoneum is covered by a mesothelial monolayer beneath which is a basement membrane and submesothelial layer that contains collagen, fibroblasts, adipose tissue, blood vessels and lymphatics. During peritoneal dialysis (PD), peritoneal cells are repeatedly exposed to a non-physiological hypertonic environment with high glucose content and low $\mathrm{pH}$. Mesothelial cells (MCs) play an important role in regulating the inflammatory response in the peritoneal cavity: they produce pro-inflammatory cytokines and chemoattractants. By secreting these chemokines or cytokines, MCs contribute to the recruitment of leukocytes following the expression of adhesion molecules. Chronic changes in the peritoneum with fibrosis develop after years of $\mathrm{PD}$. The most marked changes are noted in cases of severe and recurrent peritonitis. Others have made similar observations, i.e. long-term exposure to $\mathrm{PD}$ solutions appears to increase fibrosis and the probability of ultrafiltration failure.

Conventional PD fluids (PDFs) make use of the osmotic gradient generated by glucose. Years of exposure to PDFs result in the formation of an avascular layer of interstitial matrix and plasma proteins in the submesothelial compact zone and an epithelial-to-mesenchymal transition (EMT) of MCs. The fibrotic process in the peritoneal membrane develops following acute and chronic release of PD-related inflammatory mediators. Independent extrinsic and intrinsic events (table 1) contribute to chronic inflammation in patients on PD leading to complications including peritoneal membrane ultrafiltration failure, fluid overload, protein energy wasting and even atherosclerosis.

\section{KARGER \\ Fax +4161306 1234 E-Mail karger@karger.ch} www.karger.com

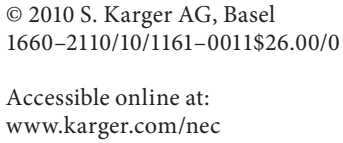

Prof. K.N. Lai

Department of Medicine, University of Hong Kong

Queen Mary Hospital

102 Pokfulam Road, Hong Kong, SAR (China)

Tel. +852 2255 4251, Fax +852 2816 2863, E-Mail knlai@hkucc.hku.hk 
Table 1. Events promoting chronic inflammation in PD

Extrinsic factors
Uremia
PDFs
Infections - especially peritonitis
Intrinsic factors
Mesothelium
Submesothelial compact zone
Submesothelial blood vessels
EMT
Receptors for GDPs and AGE
Macrophages

\section{Inflammation in PD}

Sustained low-grade systemic inflammation is highly prevalent in chronic kidney disease even at early stages. The inflammatory status of chronic kidney disease per se is affected by reduced renal clearance of cytokines, fluid overload, immunological dysfunction and infectious comorbidity. Furthermore, renal replacement therapy may also promote inflammation, e.g. in cases of dialysis access infections, bio-incompatibility of membranes or solutions and dialysate contamination [1]. In addition, genetic variations and dialysis inadequacy may also predispose some dialysis patients to an exaggerated inflammatory response $[2,3]$. While initiation of hemodialysis may partially reduce C-reactive protein (CRP) levels, this was not observed during PD initiation, suggesting different effects of individual dialysis techniques on the inflammatory outcome [4]. Regardless of the differences in techniques, inflammation in PD patients is associated with a poor prognosis, with accelerated atherosclerosis, and an increase in cardiovascular morbidity and mortality [5]. The atherosclerosis is associated with increased arterial stiffness, endothelial dysfunction and elevated oxidative stress and inflammation. A close association between hyperleptinemia, inflammation and malnourishment occurs in PD-related peritonitis [6].

\section{Extrinsic Factors}

\section{Peritoneal Dialysis Fluids}

D-Glucose is a reactive compound that exerts an effect on MCs directly by up-regulating the synthesis of transforming growth factor (TGF- $\beta$ ) and connective tissue growth factor by MCs or through its degradation path- way into glucose degradation products (GDPs) and formation of advanced glycation end-products (AGEs). Exposure to GDPs leads to enhanced cytotoxic damage and pro-inflammatory responses in MCs stimulating the production of vascular endothelial growth factor (VEGF) that enhances vascular permeability and angiogenesis. GDPs also down-regulate the expression of intercellular tight junction proteins like ZO-1, occludine and claudin-1 in MCs, again via VEGF [7].

Factors such as the buffer, glucose or GDPs formed during heat sterilization are critical in determining the biocompatibility of different PDFs. MC repair (remesothelialization) after exposure to GDPs is impaired, independent of D-glucose concentration. After exposure of mesenchymal cells to PDFs, the expression of cytokeratin 18 and E-cadherin is reduced while the expression of $\alpha$ smooth muscle actin and vimentin as a sign of EMT is increased. Expression of intercellular tight junction proteins is down-regulated after incubation with PDFs.

\section{Infection}

Bacterial peritonitis was associated with a sharp increase in total cell and neutrophil counts (400-fold) in PDFs up to 3 weeks after peritonitis despite clinical remission. There was a progressive increase in the percentage of MCs or dead cells in the total cell population in PDFs over the entire 6-week period. Dialysate levels of interleukin (IL)-1, IL-6, and TGF- $\beta$ increased markedly on day 1 before their levels decreased gradually. There is an active release of pro-inflammatory cytokines and sclerogenic growth factors for at least 6 weeks despite apparent clinical remission of peritonitis. The peritoneal cytokine networks after peritonitis may potentially affect the physiological properties of the peritoneal membrane [8].

\section{Uremia}

It has been shown that the peritoneum of uremic and current hemodialysis patients who have never been exposed to PD is abnormal as well; this finding implies that uremia induces inflammation in the peritoneum. There is a marked increase in vasculopathy below the compact zone.

\section{Plasticizers}

There are preliminary data demonstrating a better preservation of in vitro phagocyte function with adaptation of $\mathrm{pH}$ and reduction of plasticizers in PDFs [9]. Nevertheless, the Blue Ribbon Panel failed to collect enough evidence of harmful health effects to remove plasticizers 
from use in medical tubing. Another analysis concurred with the Panel's recommendation that alternative materials be developed and studied [10].

\section{Intrinsic Factors}

\section{Mesothelium}

Peritoneal MCs are biologically active and play distinctive biological roles other than local host defense [7]. The MCs are sensitive to the effect of $\mathrm{pH}$ although conventional PDFs are usually buffered from $\mathrm{pH} 5.2$ to 7.4 for $15-30 \mathrm{~min}$ in clinical studies while TGF- $\beta$ production by MCs is less with bicarbonate-buffered PDF. Glucose in the PDF can bring about major changes in the environment of MCs as well as that of the cells underlying the mesothelium, and production of various cytokines is increased as a result of this exposure. It is noteworthy that glucose may exert little effect on the synthesis of specific mediators, such as VEGF, yet its synthesis is greatly enhanced by GDPs or AGE. A serial peritoneal biopsy study showed denudation of the mesothelial monolayer as early as 6 months after maintenance PD.

\section{Submesothelial Compact Zone}

After years of continuous $\mathrm{PD}$, a good percentage of patients would have marked increase in the thickness of the submesothelial compact zone. The layer resembles scar tissue with a relatively amorphous, avascular appearance. Animal studies reveal that a spotty inflammation is detected at different places of the peritoneum in the first few weeks of PDF exposure. With time, these areas of inflammation and sclerosis gradually coalesce and become more uniform to cover much of the peritoneum that is in contact with the PDF. As the fibrosis becomes more uniform, the patient will gradually lose ultrafiltration. An avascular layer in the submesothelial compact zone results in a marked decrease in the concentration of osmotic agents in the vicinity of the filtering vasculature. An avascular matrix of macromolecules is laid down over the abnormal microvasculature, which includes vessels produced by angiogenesis. The glucose in the PDF will diffuse down its concentration gradient through the avascular gel matrix to the blood vessel underlying this matrix. Uptake of glucose by these vessels will be quite rapid following increased permeability due to abnormal angiogenic vessels and the increased surface area of the microvasculature. Diffusion through such a matrix will occur rapidly as well, due to the low resistance of the abnormal interstitium and the lack of cells. Thus the glucose gradi- ent or osmotic gradient between the interstitium and the blood vessels could be rapidly dissipated. The osmotic filtration from the abnormal blood vessels into the interstitium will likely occur at a low rate because of the reduced osmotic force adjacent to the microvasculature. The filtered fluid, once it leaves the blood and migrates into the tissue, has no means to move from the deeper interstitium into the peritoneal cavity. In addition, fibrosis speeds the absorption of glucose into the gel matrix and ultimately into the blood, and effectively lowers the osmotic pressure adjacent to the blood vessels, hence eliminating the osmotic filtration dramatically.

\section{Submesothelial Blood Vessels}

In parallel with fibrosis, the peritoneum shows a progressive increase in capillary number (angiogenesis) and vasculopathy, which are involved in both the elevation of small solute transport across the peritoneal membrane and ultrafiltration failure. GDPs stimulate VEGF production by MCs. Local VEGF production during PD appears to play a central role in the processes leading to peritoneal neoangiogenesis and functional decline. The changes in the structure of the peritoneal function over time on PD, as found in functional tests, has been confirmed in biopsy studies performed on patients. These show both neoangiogenesis and fibrosis as the underlying morphological changes contributing to these phenomena. As mentioned previously, glucose uptake by submesothelial blood vessels will be quite rapid following increased permeability due to abnormal angiogenic vessels and the increased surface area of the microvasculature. This results in dissipation of the osmotic driving force through increased area and solute transport. In addition, disruption of intercellular tight junctions in MCs may occur following down-regulation of ZO-1 expression, in which VEGF plays an important role [7].

\section{Epithelial-to-Mesenchymal Transition}

Chronic exposure of the mesothelium to sterile PDFs results in an EMT. Local inflammation and oxidative stress, which results from the continuous peritoneal injury, accelerate the EMT of peritoneal MCs resulting in peritoneal fibrosis and ultrafiltration failure. EMT is a process by which the MCs undergo a progressive loss of the epithelial phenotype and acquire fibroblast-like characteristics, which allows these cells to invade the mesothelial stroma contributing to angiogenesis, fibrosis and ultrafiltration failure. Yanez-Mo et al. [11] recovered and cultured human MCs from the spent dialysate of 54 stable patients. Eighty-five percent of these patients had no pre- 
vious peritonitis. Omental fibroblasts were separated from 3 omental MC samples from 39 patients on continuous ambulatory PD. There was a transition from an epithelial type of MC to a fibroblast-like cell with loss of normal markers of the mesothelium and phenotypic changes following progressive and continuous exposure to PDFs. For patients who were exposed to dialysate for more than 12 months, their MCs changed from $75 \%$ cobblestone phenotype to less than $30 \%$ with the remainder being fibroblast like. In some patients they observed that in less than 9 months there was loss of cytokeratin in the MC layer. These findings suggest chronic exposure of the peritoneum to the current glucose-based PDF leads to morphologic and phenotypic changes in the mesothelium with 24 months.

TGF- $\beta$, more specifically TGF- $\beta_{1}$, is one of the main mediators of the profibrotic effects of $\mathrm{PD}$ solutions through the Smad 2 and 3 pathways. These effects include fibroblast activation, collagen deposition, inhibition of fibrinolysis, maintenance of fibrosis and neoangiogenesis [7]. Acting through the Smad pathway, TGF- $\beta$ induces $\beta$-catenin formation, which in conjunction with activator protein-1 activates matrix metalloproteinase- 9 expression facilitating the invasion of the extracellular matrix [12]. Interestingly, angiotensin II inhibitors (which suppress TGF- $\beta$ activity) have recently been shown to reduce peritoneal fibrosis and neoangiogenesis, as well as to prevent the increase in small solute transport in longterm PD patients [13]. Non-viral microbubble delivery of the Smad 7 transgene markedly abolishes the peritoneal fibrosis induced by glucose-containing PDF [14]. Neutrophil gelatinase-associated lipocalin (NGAL) is specifically induced in human peritoneal MCs by IL-1 $\beta$. Lately, Leung et al. [15] demonstrated that incubation of human peritoneal MCs with recombinant NGAL reversed the TGF- $\beta$-induced up-regulation of Snail and vimentin but rescued the down-regulation of E-cadherin. Their data suggest that NGAL may exert a protective effect in modulating the EMT activated following peritonitis.

\section{Receptors for GDPs and AGE}

AGEs have been detected immunohistochemically in the peritoneum of PD patients. Receptor for advanced glycation end-products (RAGE) is the best-characterized signal transduction receptor for AGEs. Primarily binding of AGEs to their receptor was regarded as a scavenger receptor involved in AGE removal and clearance. However, ligand binding to RAGE results in an activation of key signal transduction pathways, such as NF- $\kappa \mathrm{B}$, and multiple cellular signaling cascades, e.g. activation of mito- gen-activated protein kinase. Local interaction between RAGE and AGEs/GDPs leads to the development of peritoneal inflammation, neoangiogenesis and, finally, fibrosis. Anti-RAGE antibody partially prevents the development of submesothelial and interstitial fibrosis and EMT in an animal model of peritoneal fibrosis [16]. Aminoguanidine (AG) prevents AGE formation. Supplementation of AG to PDF showed inhibitory effects on peritoneal AGE accumulation, mesothelial denudation, submesothelial monocyte infiltration, peritoneal permeability and ultrafiltration, and preserved the functional capacity of peritoneal macrophages in the rat. PDF-induced fibrosis was significantly reduced by AG [17]. The use of AG in human is limited by its $\mathrm{pH}$ and toxicity.

It is now evident that RAGE is much more than a single receptor for AGEs or a scavenger receptor: it has a broad repertoire of ligands. The key pathophysiological step seems to be GDP-dependent AGE formation in the uremic milieu, through which enhanced RAGE expression could be observed in the peritoneum. Recently, other AGE receptors, including AGE-R-1 (p 60), AGE-R-2 (p 90) and AGE-R-3 (gallectin-3) have also found to be expressed on MCs [18]. Different GDPs exert differential regulation and expression of these receptors on human peritoneal MCs [18]. However, the functional significance of these various forms has not yet been completely delineated.

\section{Macrophages}

Resident macrophages increase markedly with bacterial peritonitis and are able to enhance the release of peroxide and pro-inflammatory cytokines, including IL-1 and TNF- $\alpha$. TGF- $\beta$ complementary DNA (cDNA) molecules per macrophage are significantly greater than those of macrophages in non-infective PDFs throughout the peritonitis period [8]. There was no significant correlation between PDF levels of TGF- $\beta$ and TGF- $\beta$ cDNA molecules per macrophage, suggesting that peritoneal macrophages are not the only source of TGF- $\beta$ in PDFs.

\section{Encapsulating Peritoneal Sclerosis}

Encapsulating peritoneal sclerosis (EPS) is a devastating complication of PD thought to result from chronic intra-abdominal inflammation that is multifactorial in origin. EPS is uncommon - incidences are $0.8-2.5 \%$ in Japan and $1.5 \%$ in Scotland. Prolonged PD represents the most consistent 'risk factor' identified. One of the most characteristic pathologic findings in EPS is peritoneal fi- 
brosis. In that respect, peritoneal MCs and fibroblasts play important roles in collagen production through the expression of cytokines and growth factors. Several recent studies have delineated many new aspects of peritoneal cell biology and immunology, in terms of fibrosis and inflammation alike. Hence, factors mentioned previously in chronic inflammation, e.g. TGF- $\beta$ and IL-1, and uremia are operative in the development of EPS. In addition, Toll-like receptors and heat shock protein 47 play contributory roles in EPS [19].

\section{The 'Less-Recognized' Inflammatory Role of Peritoneal Adipocytes in PD}

Peritoneal adipocytes, previously viewed as less important in peritoneal physiology during $\mathrm{PD}$, are ubiquitously found in all peritoneal tissue. Adipose tissue is abundant in omental or mesenteric peritoneum but less so in parietal, intestinal and diaphragmatic peritoneum. In parietal peritoneum, adipocytes lie deeply under the mesothelium and connective tissue. During the fluid dwell in PD, solutes in PDF move by passive diffusion through the peritoneal barrier and come into contact with the adipocytes. Ultrastructural study reveals that a portion of the adipocyte protrudes from the mesothelial surface, thus omental adipocytes may come into direct contact with dialysate. In addition, dialysate may also reach the parietal adipose tissue when there is junctional damage or denudation of the mesothelial monolayer. It is therefore logical to postulate that with repeated exposure to PDF and the continuous changes in peritoneal physiology during maintenance $\mathrm{PD}$, peritoneal adipocytes will inevitably be 'activated'.

There is now compelling evidence that adipocytes can mediate various physiological processes through the secretion of an array of adipokines that include leptin, adiponectin, resistin, TNF- $\alpha$, IL- 6 , TGF- $\beta$ and other growth factors. Moreover, adipocytes express receptors for leptin, insulin-like growth factor-1, TNF- $\alpha$, IL-6 and TGF- $\beta$, and may form a network of local autocrine, paracrine and endocrine signals [20]. In PD patients, this is of special significance, as the initiation of treatment is often associated with an increase in fat mass that could be associated with the genetic effect on energy metabolism in addition to glucose absorption from the PDF. Interestingly, there is a difference in the release of growth factors between visceral and subcutaneous adipose tissue [21]. The omental adipose tissue most affected by PD releases $2-3$ times more IL- 6 than the subcutaneous fat tissue. Visceral fat mass correlates significantly with circulating IL-6 levels but not for non-visceral fat mass. Human subcutaneous adipocytes secrete potent mineralocorticoid-releasing factors, suggesting a direct link between obesity and hypertension [22]. Similar study in peritoneal adipocytes is lacking.

Lately, the potential inflammatory role of peritoneal adipocytes has been suggested by novel data on leptin synthesis and leptin receptor expression in peritoneal tissue. Both mRNA and protein of the full-length leptin receptor are found to be constitutively expressed in human peritoneal MCs [23]. Leptin receptor expression in MCs is up-regulated by glucose but not leptin. In adipocytes, glucose increases mRNA expression and synthesis of leptin. The Janus kinase-signal transducers and activation (JAK-STAT) signal transduction pathway in MCs is activated by either exogenous or adipocyte-derived leptin. Exogenous leptin induces the release of TGF- $\beta$ by MCs. TGF- $\beta$ synthesis induced by leptin is amplified by glucose through increased leptin receptor expression. These novel findings strongly point to the notion that peritoneal adipocytes activated by the PDF exert pro-inflammatory effects on MCs through the release of adipokines and hence contribute to altered peritoneal physiology and dysfunction during PD.

\section{Clinical Syndrome of Chronic Inflammation in PD}

The above-mentioned dialysis risk factors and certain PD-specific characteristics are associated with the inflammatory burden possibly linking inflammation, increased peritoneal solute transport rate and declined residual renal function to poor outcome. Both local (intraperitoneal) and systemic inflammation may additively be the cause and consequence of peritoneal membrane failure, and are important prognosticators of mortality in PD patients. Several factors deserve special emphasis. It has been shown that even with apparent clinical remission of PD-related peritonitis, dialysis patients, after an episode of peritonitis, may still be affected by prolonged systemic chronic inflammation. The significantly prolonged inflammation contributed to a poorer nutritional status and higher mortality [6]. The finding is consistent with our previous study that the level of cytokines in the peritoneal effluent remained higher than that in non-infective effluent throughout the 6-week period after peritonitis in parallel with elevated serum CRP, despite clinical remission [8]. One sixth of these patients with prolonged elevation in serum CRP died of a cardiovascular 


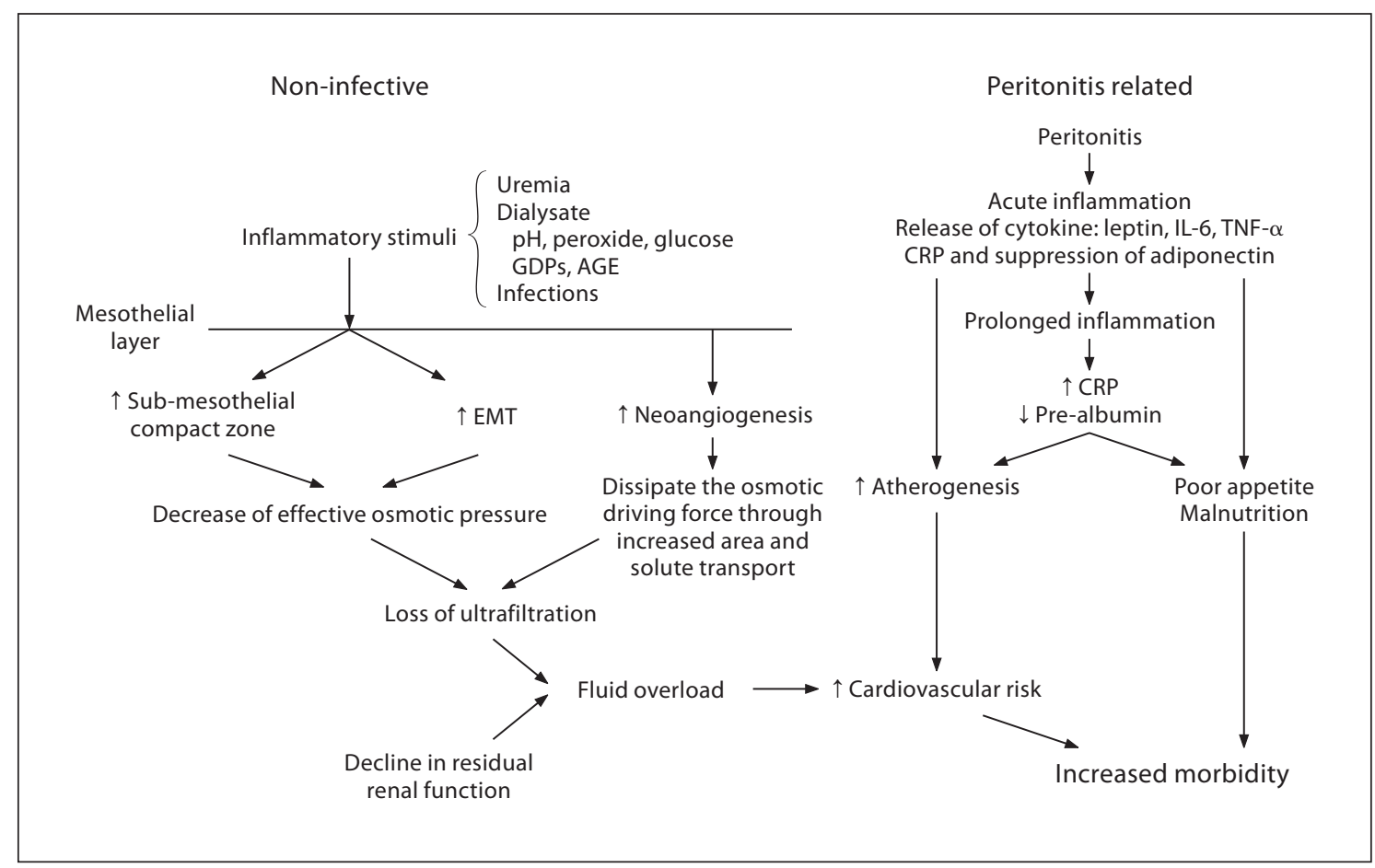

Fig. 1. Interactions between peritonitis-related and non-infective factors leading to chronic inflammation and increased morbidity in PD patients.

Fig. 2. Different practical and potential therapeutic options to minimize chronic inflammation in $\mathrm{PD}$ patients. $\mathrm{MAP}=\mathrm{Mi}-$ togen-activated protein; $\mathrm{PKC}=$ protein $\mathrm{ki}-$ nase $\mathrm{C}$.

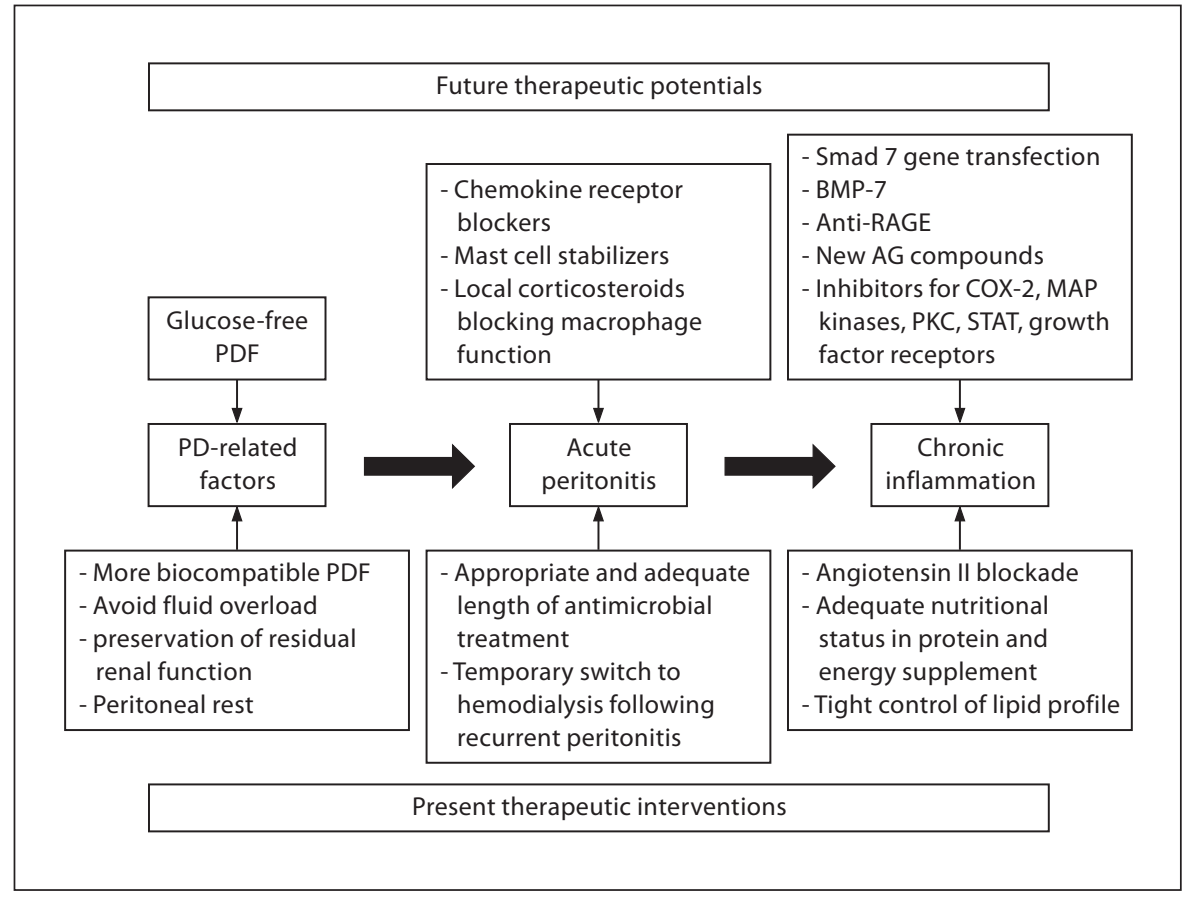


event over a median period of 17 months [24]. Therefore, prolonged inflammation is likely to potentiate atherogenesis and increase the risk of cardiovascular events.

Other than persistent low-grade inflammation, subclinical malnutrition may be another factor for the high mortality in these patients. Chronic inflammation with atherosclerosis is closely related to malnutrition, forming the malnutrition-inflammation-atherosclerosis syndrome [24]. The underlying mechanism for malnourishment is likely to be multifactorial. Possible contributory factors include protein loss in the dialysate, the feeling of fullness due to PDF in the abdomen, uremia-associated cachexia caused by leptin signaling through the hypothalamic melanocortin receptor [25], and protein energy wasting. The complications of membrane failure and fluid overload further enhance a higher incidence of cardiovascular events. Our proposal of a hypothetical mechanism of chronic inflammation in PD is shown in figure 1 .

\section{Newer Osmotic Agents in PDF}

Low GDP solutions clearly have an advantage over high GDP solutions [26]. But the continued presence of glucose remains a significant problem for the cells. Alter- native hypertonic agents with additive that may prevent chronic inflammation will continue to be a subject of research. The issue of mechanical stress effects from the large volume of any solution in the cavity, which might induce chronic changes in the peritoneum, remains a major concern.

\section{Conclusion}

Attention for chronic inflammation should be practiced in PD patients, especially following peritonitis. Adequate nutritional support and screening for persistent inflammation are warranted such that the vicious circle (malnutrition-inflammation-atherosclerosis syndrome) can be abolished. Figure 2 shows possibilities for therapeutic intervention to minimize chronic inflammation in PD at different levels.

\section{Acknowledgment}

Part of the work described in this paper was supported by the Seeding Fund for Basic Research, University of Hong Kong (grant No. 10203727.05485. 20600.323.01), the L. and T. Charitable Foundation and the House of INDOCAFE.

\section{References}

1 Stenvinkel P, Alvestrand A: Inflammation in end-stage renal disease: sources, consequences, and therapy. Semin Dial 2002;15: 329-337.

-2 Liu Y, Berthier-Schaad Y, Plantinga L, Fink NE, Tracy RP, Kao WH, Klag MJ, Smith MW, Coresh J: Functional variants in the lymphotoxin-alpha gene predict cardiovascular disease in dialysis patients. J Am Soc Nephrol 2006; 17:3158-3166.

- 3 Ayus JC, Mizani MR, Achinger SG, Thadhani R, Go AS, Lee S: Effects of short daily versus conventional hemodialysis on left ventricular hypertrophy and inflammatory markers: a prospective, controlled study. J Am Soc Nephrol 2005;16:2778-2788.

$\checkmark 4$ Carrero JJ, Axelsson J, Avesani CM, Heimburger O, Lindholm B, Stenvinkel P: Being an inflamed peritoneal dialysis patient: a Dante's journey; in Ronco S, Dell'Aquila R, Rodghiero MP (eds): Peritoneal Dialysis: A Clinical Update. Contrib Nephrol. Basel, Karger, 2006, vol 150, pp 144-151.

-5 Fassett RG, Driver R, Healy H, Ranganathan D, Ratanjee S, Robertson IK, Geraghty DP, Sharman JE, Coombes JS: Comparison of markers of oxidative stress, inflammation and arterial stiffness between incident hemodialysis and peritoneal dialysis patients - an observational study. BMC Nephrol 2009;10:8.

6 Lam MF, Leung JC, Lo WK, Tam S, Mong MC, Lui SL, Tse KC, Chan TM, Lai KN: Hyperleptinaemia and chronic inflammation after peritonitis predicts poor nutritional status and mortality in patients on peritoneal dialysis. Nephrol Dial Transplant 2007;22: 1445-1450.

-7 Lai KN, Tang SC, Leung JC: Mediators of inflammation and fibrosis. Perit Dial Int 2007; 27(suppl 2):S65-S71.

$\checkmark 8$ Lai KN, Lai KB, Chan TM, Lam CW, Li FK, Leung JCK: Changes of cytokine profile during peritonitis in patients on continuous ambulatory peritoneal dialysis. Am J Kidney Dis 2000;35:644-652.

9 Alscher DM, Pauli-Magnus C, Kirchgessner J, Kuhlmann U, Mettang T: A new lactatebased, plasticizer-free, neutral peritoneal dialysis fluid provided in a two-compartment system: effect on peripheral leukocyte function. Nephron 2000;86:62-69.
10 Hill SS, Shaw BR, Wu AH: The clinical effects of plasticizers, antioxidants, and other contaminants in medical polyvinylchloride tubing during respiratory and non-respiratory exposure. Clin Chim Acta 2001;304:1-8.

11 Yanez-Mo M, Lara-Pezzi E, Selgas R, Ramirez-Huesca M, Dominguez-Jimenez C, Jimenez-Heffernan JA, Aguilera A, SánchezTomero JA, Bajo MA, Alvarez V, Castro MA, del Peso G, Cirujeda A, Gamallo C, SánchezMadrid F, López-Cabrera M: Peritoneal dialysis and epithelial-to-mesenchymal transition of mesothelial cells. N Engl J Med 2003; 348:403-413

12 Selgas R, Bajo A, Jiménez-Heffernan JA, Sánchez-Tomero JA, Del Peso G, Aguilera A, López-Cabrera M: Epithelial-to-mesenchymal transition of the mesothelial cell - its role in the response of the peritoneum to dialysis. Nephrol Dial Transplant 2006;21(sup$\mathrm{pl} 2)$ :S2-S7.

13 Kolesnyk I, Noordzij M, Dekker FW, Boeschoten EW, Krediet RT: A positive effect of aII inhibitors on peritoneal membrane function in long-term PD patients. Nephrol Dial Transplant 2009;24:272-277. 
14 Guo H, Leung JC, Lam MF, Chan LY, Tsang AW, Lan HY, Lai KN: Smad7 transgene attenuates peritoneal fibrosis in uremic rats treated with peritoneal dialysis. J Am Soc Nephrol 2007; 18:2689-2703.

-15 Leung JC, Lam MF, Tang SC, Chan LY, Tam KY, Yip TP, Lai KN: Roles of neutrophil gelatinase-associated lipocalin in continuous ambulatory peritoneal dialysis-related peritonitis. J Clin Immunol 2009;29:365-378.

-16 De Vriese AS, Tilton RG, Mortier S, Lameire NH: Myofibroblast transdifferentiation of mesothelial cells is mediated by RAGE and contributes to peritoneal fibrosis in uraemia. Nephrol Dial Transplant 2006;21:25492555.

-17 Zareie M, Tangelder GJ, ter Wee PM, Hekking LH, van Lambalgen AA, Keuning ED, Schadee-Eestermans IL, Schalkwijk CG, Beelen RH, van den Born J: Beneficial effects of aminoguanidine on peritoneal microcirculation and tissue remodelling in a rat model of PD. Nephrol Dial Transplant 2005;20: 2783-2792.
$>18$ Lai KN, Leung JC, Chan LY, Li FF, Tang SC, Lam MF, Lam MF, Tse KC, Yip TP, Chan TM, Wieslander A, Vlassara H: Differential expression of receptors for advanced glycation end-products in peritoneal mesothelial cells exposed to glucose degradation products. Clin Exp Immunol 2004;138:466-475.

19 Miyazaki M, Yuzawa Y: The role of peritoneal fibrosis in encapsulating peritoneal sclerosis. Perit Dial Int 2005;25(suppl 4):S48S56.

20 Myers MG Jr: Leptin receptor signaling and the regulation of mammalian physiology. Recent Prog Horm Res 2004;59:287-304.

21 Fain JN, Madan AK, Hiler ML, Cheema P, Bahouth SW: Comparison of the release of adipokines by adipose tissue, adipose tissue matrix, and adipocytes from visceral and subcutaneous abdominal adipose tissues of obese humans. Endocrinology 2004; 145: 2273-2282.

-22 Ehrhart-Bornstein M, Lamounier-Zepter V, Schraven A, Langenbach J, Willenberg HS, Barthel A, Hauner H, McCann SM, Scherbaum WA, Bornstein SR: Human adipocytes secrete mineralocorticoid-releasing factors. Proc Natl Acad Sci USA 2003;100: 14211-14216.
23 Leung JC, Chan LY, Tang SC, Chu KM, Lai $\mathrm{KN}$ : Leptin induces TGF-beta synthesis through functional leptin receptor expressed by human peritoneal mesothelial cell. Kidney Int 2006;69:2078-2086.

-24 Stenvinkel P, Heimburger O, Lindholm B, Kaysen GA, Bergstrom J: Are there two types of malnutrition in chronic renal failure? Evidence for relationships between malnutrition, inflammation and atherosclerosis (MIA syndrome). Nephrol Dial Transplant 2000;15:953-960.

25 Cheung W, Yu PX, Little BM, Cone RD, Marks DL, Mak RH: Role of leptin and melanocortin signaling in uremia-associated cachexia. J Clin Invest 2005;115:1659-1665.

-26 Flessner MF: Sterile solutions and peritoneal inflammation; in Ronco S, Dell'Aquila R, Rodghiero MP (eds): Peritoneal Dialysis: A Clinical Update. Basel, Karger, Contrib Nephrol 2006;150:156-165.

\title{
Editorial Comment
}

\author{
M. El Nahas, Sheffield
}

Lai and Leung review the range of responses to continuous ambulatory $\mathrm{PD}$, highlighting the capacity of MCs to release a range of pro-inflammatory as well as pro-fibrotic mediators. They also emphasize the range of inflammatory, fibrotic as well as vascular (angiogenic) changes taking place within the peritoneum of patients exposed to glucose-containing dialysis solutions through the formation of AGE and GDP. Experimental data also show the capacity of MCs to transform into myofibroblasts thus contributing to fibrogenesis and peritoneal sclerosis. The authors review conditions derived from peritoneal inflammation including EPS. This serious condition is likely to be underdiagnosed, and increased awareness of its complications in patients on continuous ambulatory PD is warranted (for a comprehensive review of EPS, see Augustine et al. [1] in Nephron).

\section{Reference}

Augustine T, Brown PW, Davies SD, Summers AM, Wilkie ME: Encapsulating peritoneal sclerosis: clinical significance and implications. Nephron Clin Pract 2009;111:c149-c154. 